\title{
Les grands patrons français et la crise financière
}

FRANÇOIS-XAVIER DUDOUET, sociologie politique et morale, chercheur au CNRS (IRISSOuniversité Paris Dauphine).

ÉRIC GRÉMONT,
président de
l'Observatoire
politico-économique
des structures
du capitalisme
(OpesC), spécialiste
de l'économie
d'entreprise.

a crise financière a rendu plus animées et prégnantes certaines controverses publiques sur le rôle et la place des grands patrons dans les sociétés occidentales. Curieusement, si la question de leur responsabilité concernant la catastrophe a été très rapidement évacuée du débat public, celui-ci s'est focalisé sur un point en apparence anodin, les rémunérations. Comme si le débat était passé de l'essentiel à l'accessoire. Certes, sur un plan général, quelques têtes sont tombées au sommet des grandes entreprises ; certes, partout, politiques, journalistes, universitaires et même certains acteurs économiques ont plaidé pour une refondation du système financier international et des modes de rémunération. Pourtant, si l'on s'en tient aux faits, l'intervention publique a, pour l'instant, évité la refondation du système ou son apurement à la mode libérale. Si la viabilité d'un système financier privé, que ses propres lois avaient condamné sans appel, a été au cœur des interventions publiques, on est en droit de se demander si le but poursuivi implicitement n'était pas précisément la conservation des élites économiques sauvées opportunément de la faillite, en même temps que les entreprises qu'elles dirigent. La question de la crise et de son traitement par les États doit donc quitter le terrain strictement économique pour gagner celui de la sociologie politique permettant de poser la question : l'intervention des États n'a-t-elle pas eu finalement davantage pour effet de sauvegarder une certaine élite économique que d'apurer un système en crise ? Plus surprenant encore, pourquoi la question des rémunérations continue-t-elle de surnager alors que celle, fondamentale, de la responsabilité a été escamotée? 
Afin de répondre à ces questions, au moins pour la France, nous proposons d'observer les changements qui ont eu lieu à la tête des entreprises du CAC 40 depuis l'été 2007 (date de l'éclatement de la bulle dite des subprimes) et de les confronter aux caractéristiques sociologiques des grands patrons français. Ainsi, nous serons à même de mieux mesurer les effets de la crise sur cette population. Enfin, nous tenterons de montrer que les polémiques sur les rémunérations dévoilent surtout les ébranlements de l'organisation de nos sociétés salariales.

\section{Sociographie des grands patrons français durant les années 2000}

En France, comme dans la plupart des pays développés, les dirigeants de grandes entreprises sont de moins en moins souvent des propriétaires et de plus en plus des managers, c'est-à-dire des professionnels de la direction d'entreprise qui doivent moins leur position à leur capital économique qu'à d'autres types de ressources comme la trajectoire professionnelle, le portefeuille de relations ou l'origine scolaire. La figure du « directeur », que Berle et Means identifiaient dès les années $1930^{1}$, n’a cessé de se renforcer au cours du $\mathrm{XX}^{\mathrm{e}}$ siècle au point de devenir le type dominant des dirigeants de grandes entreprises. Ainsi, en 1981, sur les numéros 1 des 40 premières capitalisations de la Bourse de Paris, Bauer et Bertin-Mourot ${ }^{2}$ dénombraient $57 \%$ de managers contre $43 \%$ de patrons propriétaires, la proportion atteignant $55 \%$ contre $45 \%$ en 1986 au sommet des nationalisations ${ }^{3}$. Et cela sans compter les entreprises publiques, où par définition tous les patrons sont des directeurs non propriétaires. Si on intègre le secteur public, c'est donc la très grande majorité des grands patrons qui, dans les années 1980, sont des managers. Les privatisations vont, d'ailleurs, confirmer cette tendance, puisque la part des managers parmi les numéros 1 des entreprises cotées au CAC 40 passe de $77 \%$ en 1991 à $80 \%$ en 1997 et même $85 \%$ en 2002, en comptant les patrons étrangers ${ }^{4}$. Cette évolution des types de patrons au sein du CAC 40 livre deux enseignements. D'une part que le capitalisme familial n'a guère profité des privati-
1. A. Berle, G. Means, The Modern Corporation and Public Property, NY, Harcourt, Brace \& World, 1932.

2. M. Bauer, B. bertinMourot, Administrateurs et dirigeants du CAC 40. Des logiques sociales d'autocontrôle au cour des gouvernements d'entreprise, Puteaux, CNRS, Boyden, 1997.

3. Nous avons additionné les catégories "patrons d'État » et patrons "carrière en entreprise " pour former le groupe manager.

4. F.-X. Dudouet, É. Grémont, « Les grands patrons et l'État en France, 1981-2007 ", Sociétés contemporaines, 68, 2007. 
5. H. Joly, Diriger une grande entreprise française au $\mathrm{xx}^{\mathrm{e}}$ siècle: modes de gouvernance, trajectoire et recrutement, Mémoire inédit présenté pour l'habilitation à diriger des recherches, EHESS, 2008.

6. Il est possible que cette tendance à la dissociation soit l'expression d'un mouvement générationnel généralisé à la tête des entreprises du CAC 40 et à ce titre temporaire. sations pour accentuer, et même maintenir, sa position au sein des plus grandes entreprises. D’autre part que les « patrons d'État » ont massivement réussi leur reconversion dans le privé : la noblesse d'État s'est transformée en aristocratie des affaires.

Qu'en est-il pour les années 2000 ? La figure mythique du président-directeur général (PDG), qui fut particulièrement en vogue entre les années 1950 et $1990^{5}$, apparaît en déclin en ce début des années 2000 : 27 PDG pour 51 entreprises en 2002 contre 17 pour 47 en 2008. Le cumul des fonctions de président du conseil d'administration et de directeur général qu'incarne le PDG est de plus en plus rare ces dernières années, au profit des gouvernances bicéphales, notamment avec conseil de surveillance et directoire $^{6}$. L'essor de cette formule incite donc à développer une vision plus large des grands patrons en incluant indistinctement les PDG, les présidents du conseil, les directeurs généraux, les présidents du directoire et gérants pour les sociétés en commandite par action. Avec cette définition, on obtient une population de 136 patrons pour les entreprises cotées au CAC 40 entre 2002 et 2008. L'examen de la nationalité montre une proportion non négligeable d'étrangers (17 \%). Il s'agit d'une évolution notable par rapport aux années 1980-1990, où les patrons étrangers, comme Lindsay Owen-Jones chez L'Oréal, étaient rares. Toutefois, cette évolution ne doit pas laisser penser à une ouverture franche et massive des grands groupes français. Dans un cas (Lakshmi Mittal chez Arcelor Mittal), l'accession s'est faite à la suite d'une OPA hostile qui défraya la chronique. Mais, le plus souvent, il y a des patrons étrangers à la tête d'entreprises du CAC 40 à cause des grands rapprochements transnationaux à la fin des années 1990 et au cours des années 2000 : Aventis, EADS, Dexia, Arcelor, Alcatel-Lucent, Unibail-Rodamco. Dans quelques cas font exception cependant : Lindsay OwenJones chez L'Oréal, José-Luis Duran chez Carrefour, Ben Vervaayen chez Alcatel-Lucent ou Chris Viehbacher chez Sanofi Aventis. Les femmes sont les grandes absentes. On en dénombre seulement trois au cours de la période 2002-2008 : Élisabeth Badinter, présidente du conseil de surveillance de Publicis depuis 1996 ; Patricia Barbizet, 
présidente du conseil de surveillance de PPR entre 2001 et 2005 ; Patricia Russo, directrice générale d'Alcatel Lucent de 2006 à 2008.

Si on analyse à présent le capital scolaire des grands patrons, la très grande majorité a suivi des études supérieures (126 sur 136) ${ }^{7}$. Parmi les dix restants, trois ont un niveau bac ou inférieur et sept ne sont pas renseignés. Ce sont les diplômes universitaires qui reviennent le plus souvent $(31 \%$ des patrons sont titulaires d'un titre universitaire) mais cette hégémonie de l'université est trompeuse. Ils ne sont que 23 à être uniquement détenteurs de diplôme universitaire, dont la majorité d'étrangers (14 pour 9 Français). Parmi les 40 patrons français ayant connu les bancs de l'université, 31 ont suivi au moins une autre formation, dont très souvent une grande école. Contrairement à la plupart des autres pays, les diplômes universitaires continuent d'être faiblement valorisés par les élites économiques, au profit des formations proposées par les grandes écoles. De fait, sur 113 patrons français, 87 sont passés par une grande école ${ }^{8}$, soit $77 \%$ de l'effectif. Toutefois, si on distingue, chez les Français, les « propriétaires » des managers, alors la proportion chute à $62 \%$ pour les premiers et s'élève à $80 \%$ pour les seconds. Cette dichotomie montre que le capital économique continue de jouer à plein comme ressource légitime pour diriger un grand groupe et qu'en revanche, le capital scolaire s'avère une ressource indispensable pour les managers français sans fortune. Mais deux écoles en particulier se détachent très nettement de l'ensemble : Polytechnique et l'École nationale d'administration. Elles fournissent plus de la moitié des managers français (42\% de tous les grands patrons français). Cette hégémonie est à mettre en relation avec les grands corps de l'État ${ }^{9}$ dont ces écoles sont les principales pourvoyeuses. Les patrons issus de ces grands corps représentent $30 \%$ des managers et $26 \%$ de l'ensemble des patrons français, avec une prime particulière pour l'inspection des Finances, très présente dans la banque et l'assurance, et le corps des Mines, plus répandu dans l'industrie. Les écoles de commerce, qui étaient à peine observées dans le passé ${ }^{10}$, semblent être en plein essor en ce début des années 2000, 24 \% des patrons fran-
7. F.-X. Dudouet,

É. Grémont, Les grands patrons en France. Des privatisations à la mondialisation, Paris, Lignes de repères, 2010.

8. Le plus souvent : l’École normale supérieure, l'École nationale d'administration, Polytechnique, les Mines, les Ponts, Centrale, l’École nationale supérieure des télécommunications, HEC, l'École supérieure de commerce de Paris, l'Essec, l'Institut européen d'administration des affaires (Insead), Sciences-Po et Supelec)

9. Conseil d'État, Cour des comptes, inspection des Finances, corps des Mines, corps des Ponts et Chaussées, corps des Télécoms.

10. P. Bourdieu et M. de Saint-Martin, «Le Patronat », Actes de la recherche en sciences sociales, $\mathrm{n}^{\circ}$ 20-21, 1977 ; M. Bauer, B. BertinMourot, op. cit. 
çais sont issus d'HEC, l'Essec ou l'École supérieure de commerce de Paris, mais seulement $18 \%$ si on retranche ceux qui y ajoutent un passage par l'ENA.

$\mathrm{Au}$ terme de ce rapide tour d'horizon des propriétés sociales des dirigeants du CAC 40, on peut tirer plusieurs enseignements majeurs. Le capital scolaire des grands patrons français continue d'être largement structuré par le système des grandes écoles et les grands corps de l'État. Toutefois, on note la montée en puissance des patrons étrangers et des diplômés des grandes écoles de commerce, qui deviennent des fractions de plus en plus significatives de cette population. Mais la crise a-t-elle eu un effet sur ces grandes tendances sociologiques ? Observons les changements survenus à la tête des grandes entreprises françaises depuis l'éclatement de la bulle des « subprimes » en août 2007.

\section{La pérennité du milieu des affaires français}

Trois types de changement à la tête des grandes entreprises françaises entre l'été 2007 et l'automne 2009 sont à noter. Il y a tout d'abord les patrons sanctionnés pour des difficultés antérieures à la crise et que l'on retrouve principalement dans l'industrie. Des patrons du système bancaire font directement les frais de la crise. Enfin, il y a les successions normales à la tête des grands groupes qui touchent aussi bien la finance que l'industrie. Les grands patrons sanctionnés pour leurs résultats, José Luis Duran (Carrefour), Patricia Russo et Serge Tchuruk (Alcatel), Gérard Le Fur (Sanofi-Aventis), Frank Dangeard (Thomson), étaient déjà sur la sellette. La crise n’a, au mieux, qu'accéléré des démissions plutôt attendues. Les révocations de Christian Streiff (PSA) et François Morin (Véolia) au printemps 2009 sont, pour l'industrie, une conséquence quasi directe de la crise. Tous deux étaient à la tête d'entreprises du secteur automobile durement touchées par la raréfaction du crédit. Dans le secteur bancaire, en revanche, les changements sont clairement liés à la crise financière : Axel Miller et Pierre Richard (Dexia), Charles Milhaud (Caisses d'épargne) et Philippe Dupont (Banques populaires). Leurs établissements étaient particulièrement 
exposés. Le départ de Daniel Bouton de la Société générale est plus complexe, puisqu'il s'est fait en deux temps, d'abord en quittant la direction exécutive de la banque (mai 2008), puis la présidence du conseil d'administration (mai 2009). Surtout ce départ n’a pas été présenté comme directement lié à la crise financière, mais à l'affaire Kerviel ${ }^{11}$ (janvier 2008) et à l'émoi politico-médiatique suscité par les stock-options attribuées en mars 2009 aux dirigeants de la banque. Mais loin de recomposer en profondeur le paysage bancaire français, ces démissions ont eu pour effet de renforcer un vieil atavisme national : celui de placer à la tête des banques d'anciens inspecteurs des finances. Ainsi en est-il de Pierre Mariani (Dexia), Frédéric Oudéa (Société générale) et François Pérol (BPCE, qui est le nouvel ensemble résultant de la fusion des Caisses d'épargne et des Banques populaires). Si on ajoute BNPParibas, dirigée par deux anciens inspecteurs et AXA dont le président du directoire, et futur PDG, l'est aussi, on voit que l'essentiel du système financier français est aujourd'hui dirigé par d'anciens inspecteurs (seul parmi les grandes banques, le Crédit agricole échappe à la règle). Dans les autres entreprises, la nomination de nouveaux dirigeants depuis août 2007 relève du cycle normal des successions (Air France-KLM, AXA, EDF, Pernod-Ricard, Vallourec, Véolia) ou d'une création (Suez environnement). Parmi les nouveaux patrons nommés durant cette période, on dénombre : un patron issu du capitalisme familial mais dans une société, AXA, où il n'est pas un actionnaire significatif et dont il quittera la présidence en 2010, trois étrangers, trois Français diplômés notamment de grandes universités étrangères, deux normaliens, un ingénieur du corps des Mines, quatre polytechniciens dont un ingénieur civil des Mines, un ingénieur du corps des Ponts et un ingénieur de l'Aéronautique, enfin quatre énarques dont trois inspecteurs des finances et un conseiller d'État. On notera l'absence des diplômés des grandes écoles de commerce dans cette nouvelle promotion, si ce n'est Henri Proglio (HEC), nommé à la tête d'EDF, mais qui était déjà PDG de Véolia.

Les nominations survenues depuis le début de la crise n'ont donc pas modifié substantiellement les caractéris-
11. Du nom du trader Jérôme Kerviel, accusé d'avoir exposé frauduleusement la banque pour un montant de 50 milliards d'euros et amené celle-ci à liquider les positions avec une perte de 5,5 milliards d'euros en janvier 2008. 
tiques sociales observées antérieurement, en renforçant seulement les traits les plus saillants : absence de femmes, nette domination des patrons managers, principale provenance les grandes écoles et les grands corps, quasihégémonie des inspecteurs des finances dans la banque et l'assurance. Si la crise a fait des victimes, ce sont des individus, non les structures sociales qui résistent remarquablement. L'ébranlement de la finance internationale n'a donc pas pour l'heure conduit à une remise en cause des élites économiques qui, au contraire, voient leurs traits les plus caractéristiques se renforcer. Cette observation rejoint celle faite par la presse à propos des grandes banques américaines. Si des têtes sont tombées, les hommes sont restés les mêmes, au point que l'on peut se demander si les centaines de milliards de dollars dépensés par les États n'ont pas eu davantage pour effet de protéger le milieu des affaires que d'assainir le système financier. Les banques, tant françaises qu'américaines, ne se sontelles pas empressées de rembourser les États, à peine les beaux jours retrouvés ? Sans doute, comme l'affirme le directeur général de Goldman Sachs, pour être libres de reprendre leur politique de rémunération et proroger ainsi l'écosystème des milieux financiers.

\section{La question des rémunérations}

Ce n'est donc pas un hasard si les critiques les plus virulentes à l'égard des grands patrons concernent leur rémunération. En France, ce n'est pas nouveau. Depuis Philippe Jaffré, qui quitta Elf en 2000 avec un parachute doré de 250 millions de francs, la rémunération des grands patrons n'a pas cessé d'alimenter la chronique. Toutefois, la question a pris un relief particulier avec la crise financière, au point que certains dirigeants de banques renoncèrent plus ou moins spontanément à leur bonus pour l'exercice 2008 (BNP Paribas, Crédit agricole), voire au principal de leur rémunération (Daniel Bouton à la Société générale). Plus encore, sollicités de toutes parts, les grands patrons français donnèrent, au printemps 2009, des gages au moins formels d'autorégulation en créant le comité des sages, Association française des entreprises 
privées/Medef appelé à rendre, sous conditions, un avis consultatif sur la rémunération des dirigeants.

Mais ce qui est implicitement en cause à travers la rémunération des dirigeants, c'est leur légitimité à convertir un capital professionnel en capital économique. Tout d'abord, le fait de gagner beaucoup d'argent, même dans des proportions considérables, n'est pas, dans nos sociétés, considéré comme un mal en soi. Les artistes et les sportifs qui touchent parfois des rémunérations bien supérieures et auxquels se comparent souvent les grands patrons, ne font pas l'objet d'une condamnation sociale équivalente. Au-delà de l'argument, discutable, qui voudrait que celui qui dirige soit payé au moins autant que celui qui divertit, la comparaison est intéressante pour souligner que la capacité à percevoir telle ou telle rémunération dépend en définitive de la légitimité socialement reconnue à toucher telle ou telle portion des ressources disponibles. Or, ce que nous disent les scandales sur la rémunération des grands patrons, c'est que certains d'entre eux en tout cas, n'occupent pas, dans l'échelle des valeurs sociales, une position propre à légitimer un tel enrichissement. Car c'est bien d'enrichissement qu'il s'agit, mais un enrichissement d'un certain type, celui des patrons managers. En effet, les patrons propriétaires, par la valorisation de leur patrimoine en actions, ne sont pas soumis au même opprobre, alors qu'ils s'enrichissent bien plus. Leurs gains peuvent se chiffrer en centaines de millions d'euros par an, tandis que les managers dépassent rarement 5 millions. Mais, bien que les fortunes patrimoniales soient régulièrement dévoilées et commentées par la presse (voir les classements du magazine Challenges), elles n'ont jamais, à notre connaissance, suscité les scandales médiatiques des affaires Jaffré, Zacharias ou Bouton. Ce qui est condamné dans la rémunération des grands patrons, ce n'est donc pas l'enrichissement patrimonial, mais l'enrichissement de ceux qui, "venus de rien », économiquement parlant, en viennent à faire fortune sans jamais avoir risqué leur capital. Au contraire, ils ont bénéficié durant la plus grande partie de leur carrière d'un statut de salarié, voire, un temps, de celui de fonctionnaire.

En cela, la question de la rémunération des grands patrons managers rejoint celle des traders. Dans les deux 
12. O. Godechot, Working Rich, Salaires, bonus et appropriation $d u$ profit dans l'industrie financière, Paris, La Découverte, 2007 et O. Godechot, P. Lagneau-Ymonet, « D’un rapport salarial favorable à un autre. Les professionnels de la Bourse 19702000 », P. Beaubeau, C. Cossalter, C. Omnès, Le salariat bancaire: enjeux sociaux et pratiques de gestion, Nanterre, Presses universitaires de ParisOuest, 2009. cas, l'enrichissement n'est pas considéré comme légitime au regard des «formes normales » de l'enrichissement économique, où la fortune va à ceux qui risquent leur capital (ou l'héritent) et non à ceux qui le servent. La révolution qui est en cours et que $\mathrm{O}$. Godechot avait parfaitement identifiée avec les salariés de la finance ${ }^{12}$, réside justement dans la capacité croissante de certains agents économiques à générer des fortunes par la simple position salariale. C'est là que résiderait le scandale. La société salariale qui s'est développée en Occident depuis le XIX ${ }^{\mathrm{e}}$ siècle admettait peu ou prou l'inégalité économique, mais suivant une distinction assez nette, au moins sur le plan des représentations, entre salariés d'un côté et propriétaires du capital de l'autre. Certes, le passage d'un monde à l'autre a toujours été possible, mais l'enrichissement individuel légitime était réservé à celui qui risquait son capital. L'élite scolaire rejoignait le pouvoir et le service de l'État. Quand elle partait dans le privé, son gain restait modeste au regard des critères d'aujourd'hui. Quant à l'ensemble de la population salariée, son enrichissement suivait le rythme de la croissance économique et, surtout, passait par un relatif nivellement des revenus contribuant à développer cette large classe moyenne au fondement de la société fordiste. Or, c'est bien cette société qui en France comme ailleurs est remise en cause depuis les années 1970. Ce que nous enseignent les grands patrons managers et les traders, c'est que l'enrichissement individuel, dans des proportions considérables, peut dorénavant passer par le travail salarial, mais pour une minorité seulement. C'est cet état de fait qui semble poser problème, car cette opportunité remet en cause l'échelle de valeurs de la société salariale ou, pour le dire autrement, elle souligne son déclin. La publicité sur la rémunération des grands patrons et des traders affirme brutalement la fin de la société fordiste.

La crise financière n'a donc guère modifié la morphologie sociale des dirigeants des grandes entreprises en France. Elle a renforcé ses traits dominants en réduisant la place des femmes, en consolidant les anciens inspecteurs des finances dans les métiers de la finance et plus généralement en sanctionnant, pour l'heure, la victoire 
du capitalisme «managérial » sur le capitalisme familial. Cet essor des patrons managers, s'il n'est pas nouveau, prend une tournure toute différente avec l'explosion de leurs rémunérations qui vient buter contre le système de valeurs d'une classe moyenne en pleine crise. Les scandales relatifs à la rémunération des grands patrons ne font en définitive que souligner la difficulté à convertir légitimement un capital professionnel en capital économique. 